\title{
A New Kind of Seam Tracking System for Laser-MIG Hybrid Welding Based on Image Process
}

\author{
Xiangying Dang ${ }^{1, a}$, Li Yang ${ }^{2, b}$ \\ ${ }^{1}$ School of Information and Electronic Engineering, Xuzhou Institute of Technology, Xuzhou, China \\ 221008 \\ ${ }^{2}$ School of Mechanical and Electronic Engineering, Xuzhou Institute of Technology, Xuzhou, China \\ 221008 \\ adangpaper@163.com , blinlideyu@126.com
}

Keywords: Laser-MIG hybrid welding, Seam tracking, Image process, Fitting, Interpolation

\begin{abstract}
A kind of seam tracking system for laser-MIG hybrid welding has been designed to overcome the defects of existing laser-MIG hybrid welding equipments. Laser vision technology based on image processing has been studied. Line/arc fitting algorithm was applied to data construction for seam. Simulation tracking of seam was conducted by the helping of interpolation algorithm. Welding experiment was conducted with this new system. The results show that the clear skeleton of structured light can be get by image filtering and image refining. Fitting accuracy of line/arc fitting is within acceptable limit. Tracking accuracy of simulation tracking based on line interpolation and arc interpolation is high. Appearance of welding seam is well.
\end{abstract}

\section{Introduction}

Laser-MIG hybrid welding integrates advantages of laser heat source and MIG welding source. Not only defects of welding process by single heat source remedied, but also the characteristics of deep penetration, high speed and small deformation it has[1,2]. Fig.1 was the schematic diagram of laser-MIG welding. Laser beam shoots at the surface of work piece after focusing. The angle between MIG welding torch and work piece is 70 degree. U-axis is the centerline that the equipment of laser-MIG welding can be rotating around.

At present, studies on vision control of laser-MIG welding are focusing on welding pool basically. Shuang and others from Tsinghua University account with Zhang from Capital Iron and Steel Company studied the vision detection method of penetration status for laser-MIG hybrid welding. They realized the close-loop control of welding penetration status applied in hybrid welding [3]. A kind of seam tracking system for laser-MIG hybrid welding has been designed to improve the defects of existing laser-MIG hybrid welding equipments. This provides theoretical and experimental supports for advancing the automation of laser-MIG welding.

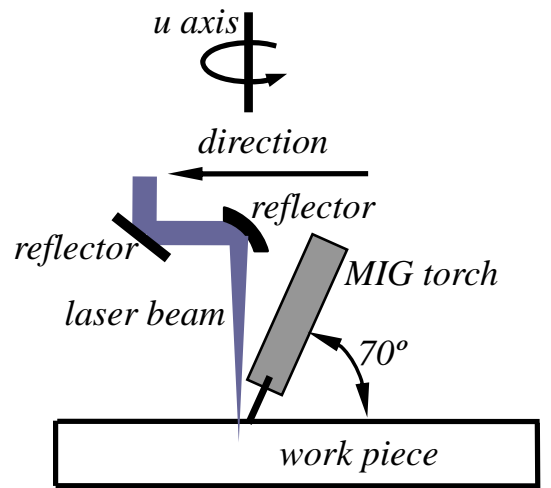

Fig. 1 Laser-MIG hybrid welding

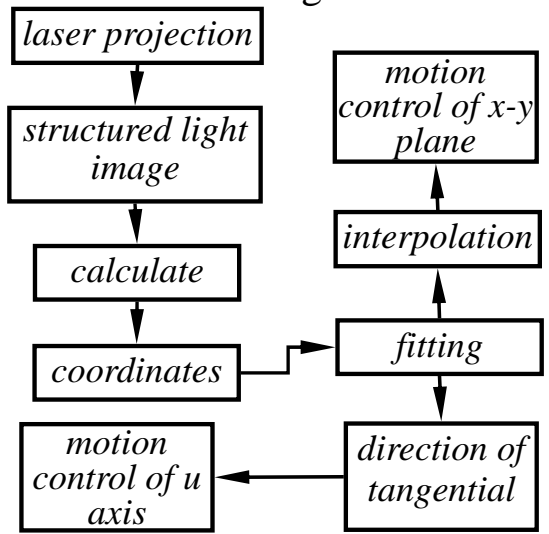

Fig. 2 Control process

\section{Design of System}

The seam tracking system for hybrid welding is realized by means of laser vision sensing. Seam 
tracking in the $\mathrm{x}-\mathrm{y}$ plane can be done by the system, also with the rotation around u-axis. Seams like line and arc can be tracked under the system. Control process is shown in Fig.2.

\section{Experiments}

Acquisition and Processing of Structured Light Image. The extraction process of structured light images is shown in Fig.3. Images were received from CCD camera by serial ports. Information of surface shape was obtained by processing of binarization, median filtering and thinning to images. The data of seam position could be got from the information [4].

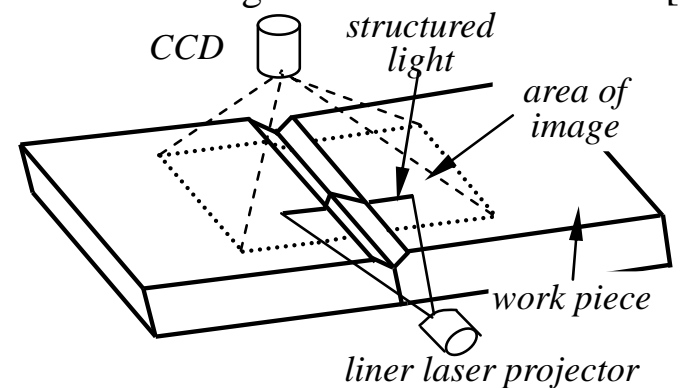

Fig. 3 Image extraction of CCD camera

Fitting of Seam and Simulation of Tracking. Coordinate data that get by processing structured light images was mapped to space coordinate. Method of approaching by line and arc was used to fitting curve section by section. Fitting process is shown in Fig.4. Sample once on every $5 \mathrm{~mm}$ in $\mathrm{x}$ direction. Arc fitting was conducted every $5 \mathrm{~cm}$. If the radius enough to $100 \mathrm{~cm}$, this section is considered as line. Line fitting will be followed. Welding piece is shown in Fig.5.Coordinates of sampling points are shown in table 1.
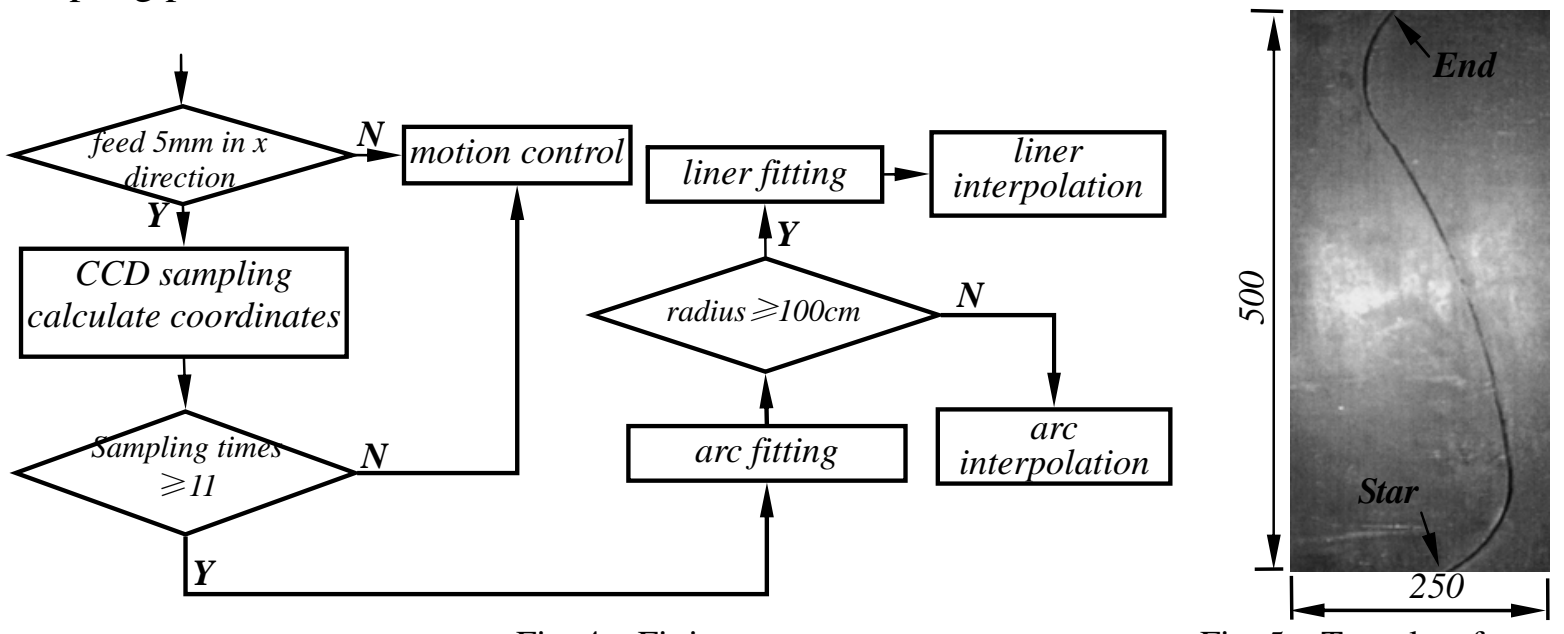

Fig. 4 Fitting process

Table 1 Coordinates of sample points [mm]

Fig. 5 Test plate for welding

\begin{tabular}{|c|c|c|c|c|c|c|c|c|c|c|c|c|c|c|c|}
\hline $\mathrm{x}$ & 1 & 6 & 11 & 16 & 21 & 26 & 31 & 36 & 41 & 46 & 51 & 56 & 61 & 66 & 71 \\
\hline $\mathrm{y}$ & 120 & 108 & 100 & 93 & 85 & 80 & 76 & 71 & 68 & 67 & 64 & 62 & 61 & 60 & 59 \\
\hline $\mathrm{x}$ & 76 & 81 & 86 & 91 & 96 & 101 & 106 & 111 & 116 & 121 & 126 & 131 & 136 & 141 & 146 \\
\hline $\mathrm{y}$ & 59 & 59 & 59 & 59 & 59 & 60 & 61 & 62 & 63 & 64 & 65 & 66 & 67 & 68 & 70 \\
\hline $\mathrm{x}$ & 151 & 156 & 161 & 166 & 171 & 176 & 181 & 186 & 191 & 196 & 201 & 206 & 211 & 216 & 231 \\
\hline $\mathrm{y}$ & 71 & 73 & 74 & 76 & 78 & 79 & 80 & 81 & 83 & 85 & 86 & 87 & 89 & 90 & 92 \\
\hline $\mathrm{x}$ & 236 & 241 & 246 & 251 & 256 & 261 & 266 & 271 & 276 & 281 & 286 & 291 & 296 & 301 & 306 \\
\hline $\mathrm{y}$ & 94 & 97 & 99 & 101 & 104 & 105 & 107 & 109 & 111 & 114 & 117 & 119 & 121 & 123 & 125 \\
\hline $\mathrm{x}$ & 311 & 316 & 321 & 326 & 331 & 336 & 341 & 346 & 351 & 356 & 361 & 366 & 371 & 376 & 381 \\
\hline $\mathrm{y}$ & 127 & 129 & 131 & 136 & 138 & 140 & 143 & 145 & 147 & 150 & 153 & 158 & 161 & 163 & 170 \\
\hline $\mathrm{x}$ & 386 & 391 & 396 & 401 & 406 & 411 & 416 & 421 & 426 & 431 & 436 & 441 & 446 & 451 & 456 \\
\hline $\mathrm{y}$ & 175 & 179 & 183 & 186 & 188 & 190 & 191 & 194 & 195 & 195 & 197 & 198 & 198 & 198 & 197 \\
\hline $\mathrm{x}$ & 461 & 466 & 471 & 476 & 481 & 486 & 491 & 496 & & & & & & & \\
\hline $\mathrm{y}$ & 195 & 195 & 193 & 191 & 189 & 187 & 184 & 182 & & & & & & & \\
\hline
\end{tabular}

The key to realize arc interpolation is arc fitting algorithm. The eleven points involved in fitting 
were numbered from 1 to 11, according to the value of $\mathrm{x}$ coordinate. To promise the continuity of welding, first point and eleventh point must locate in the arc. Error function was defined as follow.

$$
\varepsilon=\sum_{i=1}^{11} L_{i}
$$

The estimated value of radius was calculated according to the following formula.

$$
r^{2}=\left(r-D_{1}\right)^{2}+Z_{1}^{2} / 4
$$

Optimal radius was found nearby estimated value of radius. The criterion is value of $\varepsilon$ is the minimum.
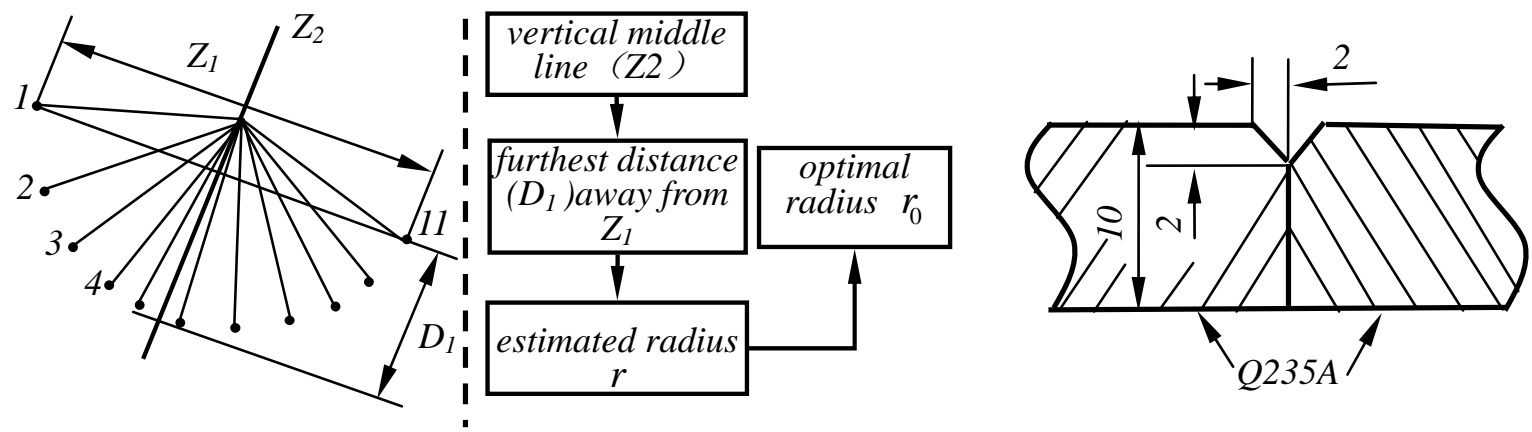

Fig. 6 Principle of fitting

Fig. 7 The schematic diagram of welded joint

Welding Experiment. Q235A steel plane with width 10mm was used as base metal. Assemble form of joint is shown in Fig.7. Curved seam is shown in Fig.5. Material of welding wire is H08A. Shielding gas of MIG welding torch is mixed gas of He and Ar. The proportion of He and Ar is 0.25. Welding procedure parameters of laser-MIG hybrid welding were shown in table 2. Two welding methods were used to test. The first one is the method that track seam by CCD camera without line/arc fitting. It samples every $2 \mathrm{~mm}$. The second one is the method that mentioned above.

Table 2 Welding procedure parameters

\begin{tabular}{|c|c|c|c|c|c|}
\hline Laser power & Welding speed & Defocusing & $\begin{array}{c}\text { Distance of heat } \\
\text { source }\end{array}$ & Welding current of MIG & Gas flow \\
\hline $5000 \mathrm{~W}$ & $1100 \mathrm{~mm} / \mathrm{min}$ & $1 \mathrm{~mm}$ & $3 \mathrm{~mm}$ & $180 \sim 200 \mathrm{~A}$ & $25 \mathrm{~L} / \mathrm{min}$ \\
\hline
\end{tabular}

\section{Results and Discussion}

Result and Discussion of Structured Light Process. Process of structured light is shown in Fig.8. Picture (a) is original picture. The picture after binarization is picture (b). Picture (c) is the picture that after median filtering. After thinning, the picture becomes picture (d). Picture (d) can reflect the surface shape information of welding seam. Coordinates of seam in the picture could be calculated. Thus, real coordinates were get by means of mapping.

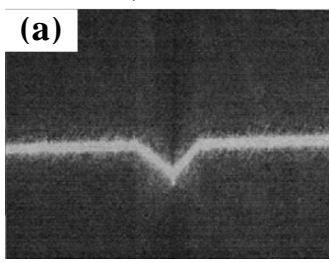

Fig. 8 Image processing for structure light

Result and Discussion of Seam Fitting. Error of every fitting point after line/arc fitting was shown in Fig.9. Maximum value of error is $0.983 \mathrm{~mm}$. Value of error is within the acceptable range according to the width of welding seam $(4 \mathrm{~mm})$. That is to say line/arc fitting is viable to seam fitting.

Result and Discussion of Interpolation. Interpolation algorithm with the accuracy of $0.01 \mathrm{~mm}$ was employed to conduct simulation track. Error of simulation is shown in Fig.10. Maximum error value is only $0.21 \mathrm{~mm}$. The result is satisfactory because that simulation of seam tracking by means of interpolating in $\mathrm{x}-\mathrm{y}$ plane.

Result and Discuss of Welding Experiment. Welding seams are shown in Fig.11. Picture (a) was is the seam welded by the method without fitting. Picture (b) is the seam welded by method 
with fitting. The seam tracking system mentioned above is superior to the traditional one in tracking accuracy. The welding appearance of welding seam is well.

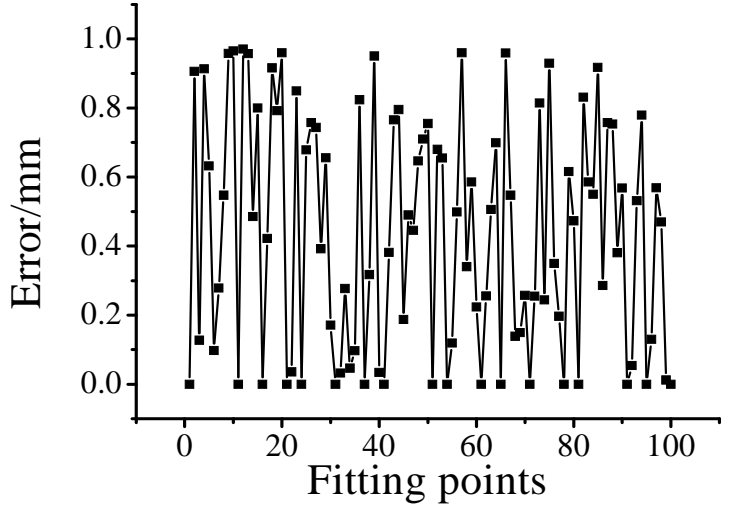

Fig. 9 Error of fitting

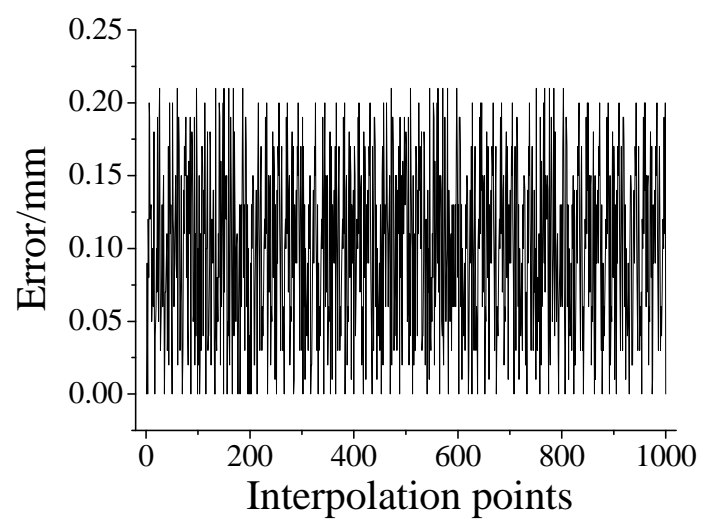

Fig. 10 Error of interpolation

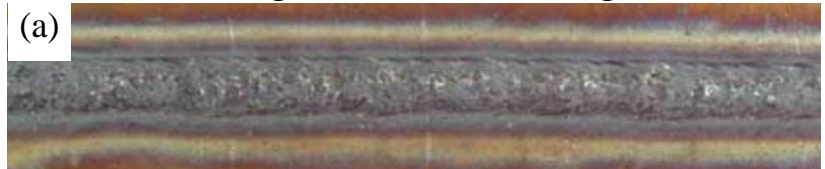

\section{(b)}

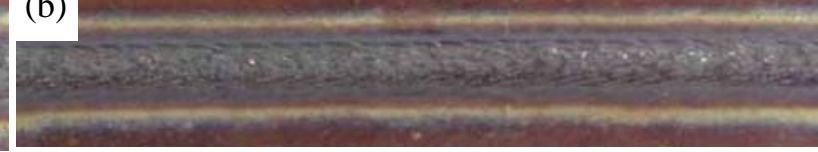

Fig. 11 Welding seams

\section{Conclusions}

1) Clear skeleton of structured light can be obtained by image refining algorithm after filtering.

2) Coordinates of seam can be got by means of vision technology. Line/arc fitting was employed to fit these sampling points. Fitting accuracy is in the scope of allowing.

3) Simulation tracking of seam was conducted by the helping of interpolation algorithm. Tracking accuracy is high.

4) The appearance of seam welded by means of the seam tracking system is well.

\section{Acknowledgements}

The authors acknowledge the financial support of this work from the Scientific and Technology Support Program of Jiangsu Province (BE2011048).

\section{References}

[1] W.M. Stun. Journal of Application Physics, Vol. 11 (1980) No.51, p.5636.

[2] C. Bagger, F.O. Olsen. Journal of Laser Applications, Vol. 17 (2005) No.1, p.2.

[3] Y.Q. Shuang, W.Z. Chen, K.J. Wang, et al. Journal of Tsinghua University (Science and Technology), Vol. 11 (2008) No.48, p.1891.

[4] L. Yang, C.C. Du, Z.Y. Zhai, et al. Hot Working Technology, Vol.40 (2011) No.23, p.168. 